\title{
A 10-Year Aortic Center Experience with Hybrid Repair of Chronic "Residual" Aortic Dissection After Type A Repair
}

\author{
Marine Gaudry $^{1,2}$ (D) Alizée Porto ${ }^{2,3}$. Arnaud Blanchard ${ }^{1,2} \cdot$ Jean-Victor Chazot ${ }^{1,2}$. Laurence Bal ${ }^{1,2}$. \\ Mariangela De Masi $^{1,2}$ - Axel Bartoli ${ }^{2,4}$. Pierre-Antoine Barral ${ }^{2,4}$ - Alexis Jacquier ${ }^{2,4}$ Vlad Gariboldi $^{2,3}$. \\ Fréderic Collart $^{2,3} \cdot$ Valérie Deplano $^{5}$ - Philippe Piquet ${ }^{1,2}$
}

Accepted: 24 January 2021

(C) Springer Science+Business Media, LLC, part of Springer Nature 2021

\begin{abstract}
Purpose Hybrid aortic arch repair in patients with chronic residual aortic dissection (RAD) is a less invasive alternative to conventional surgical treatment. The aim of this study was to describe the short-term and long-term results of hybrid treatment for RAD after type A repair.

Methods In this retrospective single-center cohort study, all patients treated for chronic RAD with hybrid aortic arch repair were included. Indications for treatment were rapid aortic growth, aortic diameter $>55 \mathrm{~mm}$, or aortic rupture.

Results Between 2009 and 2020, we performed 29 hybrid treatments for chronic RAD. Twenty-four patients were treated for complete supra-aortic debranching in zones 0 and 5 with left subclavian artery debranching alone in zone 2 . There was 1 perioperative death $(3.4 \%)$ : The patient was treated for an aortic rupture. There was no spinal cord ischemia and 1 minor stroke (3.4\%). After a median follow-up of 25.4 months (range 3-97 months), the long-term mortality was $10.3 \%$ (3/29) with no late aortic-related deaths. Twenty-seven patients (93.1\%) developed FL thrombosis of the descending thoracic aorta; the rate of aneurysmal progression on thoraco-abdominal aorta was $41.4 \%(12 / 29)$, and the rate of aortic reintervention was 34.5\% (10/29). Conclusion In a high-volume aortic center, hybrid repair of RAD is associated with good anatomical results and a low risk of perioperative morbidity and mortality, including that of patients treated in zone 0 . A redo replacement of the ascending aortic segment is sometimes necessary to provide a safer proximal landing zone and reduce the risk of type 1 endoleak after TEVAR.
\end{abstract}

Keywords Type A aortic dissection · Chronic residual type B aortic dissection · Hybrid repair · Supra-aortic debranching

Meeting presentation: European Society of Vascular Surgery (ESVS) Hamburg, September 2019

\section{Marine Gaudry}

marine.gaudry@ap-hm.fr

Valérie Deplano

valerie.deplano@univ-amu.fr

1 Department of Vascular Surgery, Timone Hospital, APHM, 13005 Marseille, France

2 Timone Aortic Center, Timone Hospital, APHM, 13005 Marseille, France

3 Department of Cardiac Surgery, Timone Hospital, APHM, 13005 Marseille, France

4 Department of Radiology, Timone Hospital, APHM, 13005 Marseille, France

5 Aix-Marseille Université, CNRS, Ecole Centrale Marseille, IRPHE UMR 7342, 13384 Marseille, France

$\begin{array}{ll}\text { Abbreviations } & \\ \text { RAD } & \text { Residual aortic dissection after type A repair } \\ \text { HR } & \text { Hybrid repair } \\ \text { TEVAR } & \text { Thoracic endovascular aortic repair } \\ \text { SA } & \text { Supra-aortic } \\ \text { IA } & \text { Innominate artery } \\ \text { LCCA } & \text { Left common carotid artery } \\ \text { LSA } & \text { Left subclavian artery } \\ \text { CPB } & \text { Cardiopulmonary bypass } \\ \text { CA } & \text { Circulatory arrest } \\ \text { ACP } & \text { Antegrade cerebral protection } \\ \text { CT } & \text { Computed tomography } \\ \text { CSFD } & \text { Cerebrospinal fluid drainage } \\ \text { STABILISE } & \text { Stent-assisted balloon-induced intimal disrup- } \\ & \text { tion and relamination in aortic dissection } \\ & \text { repair } \\ \text { FL } & \text { False lumen } \\ \text { NIRS } & \text { Near-infrared spectroscopy }\end{array}$




$\begin{array}{ll}\text { SCI } & \text { Spinal cord ischemia } \\ \text { CI } & \text { Confidence interval } \\ \text { FET } & \text { Frozen elephant trunk }\end{array}$

\section{Introduction}

The rate of reintervention after type A aortic dissection repair is estimated to be between 10 and $25 \%$ and is directly related to residual aortic dissection (RAD) in the arch and distal aorta [1-5]. These reinterventions are influenced by the technique used for the initial type A dissection repair. The avoidance of arch treatment during the initial procedure is of great concern, as the aortic arch is the most challenging area to treat, whether at the first procedure or at a later date.

Conventional open total aortic arch replacement provides durable results, but is a technically demanding redo surgery that requires prolonged cardiopulmonary bypass and circulatory arrest, leading to significant morbidity and mortality rates between 5 and $20 \%$ [6, 7]. Full endovascular techniques with branched or fenestrated endoprostheses are still in development and associated with a neurological risk ranging from 5 to $20 \%$, depending on the type of pathology [8]. Hybrid repair (HR), which is associated with thoracic endovascular aortic repair (TEVAR) and open supra-aortic (SA) debranching to provide an adequate proximal landing zone, has been introduced to address these lesions [9]. The outcomes of such hybrid aortic arch repair compared to open surgical repair are not well documented in the literature [10]. The application of HR in mostly high-risk patients increases adverse outcomes and makes the assessment of those results difficult. Outcomes of this approach seem to be directly correlated with the number of arterial debranching procedures performed as well as the proximal landing zone and the type of pathology treated.

The aim of this study was to analyze the short-term and long-term results of hybrid aortic arch repair for residual distal dissection after type A aortic repair.

\section{Patients and Methods}

\section{Study Population}

All patients included in this study were clearly informed about the use of their data for clinical research. Additionally, they were aware that their data would be used anonymously for research purposes. The institutional review board approved the project (approval number 2019-48) and waived the requirement of informed consent for the use of the patients' medical data for this study.
In this single-center retrospective cohort study, we included all patients who received hybrid treatment with a proximal landing zone of the stent graft in the aortic arch for chronic RAD. Our center is an aortic center specialized in the treatment of acute aortic syndromes. HR for aortic arch lesions is our first-line option; it is preferred over frozen elephant trunk and branched or fenestrated endoprostheses.

All cases were discussed in a multidisciplinary meeting that included vascular surgeons and cardiac surgeons, as well as an interventional radiologist, a cardiologist, and an anesthesiologist, and individualized treatment strategies were developed.

Indications for treatment were aortic rupture, rapid aortic growth (> $10 \mathrm{~mm} /$ year), or a diameter greater than $55 \mathrm{~mm}$.

Patients were included as soon as treatment of the lesion required transposition of at least one SA trunk prior to TEVAR, regardless of whether it was the innominate artery (IA), left common carotid artery (LCCA), or left subclavian artery (LSA). Endovascular treatment during type A repair was an exclusion criterion.

Demographic, anatomic, and intraoperative details were collected. Operative complication rates as well as anatomic response and reinterventions were noted during follow-up.

\section{Initial Surgery for Acute Type A Aortic Dissection}

When the entry tear was located in the ascending aorta, we performed the replacement of the ascending aorta or the hemiarch aorta. Hemiarch replacement with open distal anastomosis during moderate hypothermic circulatory arrest (CA) with cardiopulmonary bypass $(\mathrm{CPB})$ and selective antegrade cerebral perfusion (ACP) was preferred when possible (to include repair of the lesser curvature of the arch). Partial arch replacement, which was defined as a replacement between the IA and LSA, was performed in cases of primary entry tear between the IA and LSA. In these cases, the IA was debranched in the proximal part of ascending aortic repair. Total arch replacement was performed when the primary entry tear was located in the distal part of the aortic arch and could not be repaired, and when the distal anastomosis could be performed after the LSA with a frozen elephant trunk. Nevertheless, total arch replacement was seldom used in the initial acute type A repair to better enhance early survival, and partial arch replacement with IA debranching was preferred in most of cases.

Aortic root replacement with a composite prosthesis was performed according to the modified Bentall procedure in patients with dilation of the aortic root or an aortic root damaged by the entry tear.

After systemic heparinization, CPB was established by direct cannulations of the right axillary artery. Retrograde cold blood cardioplegia was infused every $15 \mathrm{~min}$. CA was instituted when the vesical temperature was $25^{\circ} \mathrm{C}$. We preferred moderate hypothermia with ACP for cerebral protection 
during CA. In all cases, bilateral invasive blood pressure monitoring was performed via radial arteries. Additionally, intraoperative cerebral monitoring was provided by near-infrared spectroscopy (NIRS) and transcranial Doppler ultrasonography.

\section{Hybrid Repair Procedure for Aneurysmal Degeneration of RAD}

Procedures were performed in two separate interventions: the debranching procedure followed by TEVAR around 1 month later.

\section{Supra-Aortic Debranching}

We used the Ishimaru classification to categorize the proximal landing zone of the stent graft.

- Zone 0 (Z0) debranching was performed by a prosthetic bypass between the ascending aortic replacement, IA, LCCA, and LSA through a median sternotomy.

The choice of a redo replacement of the ascending aortic segment was made in case of graft angulation (Fig. 1a) or a short previous ascending aortic replacement (Fig. 1b) that did not provide a good proximal landing zone.

In these cases, we performed a partial aortic arch replacement with CPB and CA with ACP to ensure a proximal landing zone $>20 \mathrm{~mm}$ without angulation (Fig. 1c-d) to prevent a type I endoleak.

The technique used to redo partial aortic arch replacement was the same as previously described for the type A repair. The choice of the cannulation technique was made preoperatively based on computed tomography (CT) scan, but right axillary artery cannulation was our preferred choice when possible. CA was performed under moderate hypothermia with ACP.

In cases of previous IA debranching (during type A repair), we performed an intra-thoracic debranching without CPB with direct or indirect reimplantation of the LCCA in young patients without comorbidities (Fig. 2a) or an extra-thoracic debranching with a carotid to carotid prosthetic bypass ( $8 \mathrm{~mm}$ prosthesis) in elderly patients with comorbidities (Fig. 2b).

- Zone 2 debranching was performed by direct or indirect LSA reimplantation through a left supraclavicular incision in cases of previous total arch replacement or when the RAD did not involve the aortic arch (Fig. 3a-b).

\section{TEVAR}

Procedures were performed in a hybrid suite (Discovery IGS 730, GE Healthcare, Chicago, USA) by both a vascular surgeon and a radiologist. Transesophageal ultrasound was used systematically to ensure the correct positioning of the guide in the true lumen. Two different stent grafts were used: C-TAG (WL Gore \& Associates Inc. Flagstaff, AZ.) and Zenith TX2 with proximal bare stent (Cook Medical, Bloomington, IN). The decision to extend the proximal landing zone was based on the location of the main residual entry tear (whether at the distal anastomosis of the ascending aortic repair, aortic arch, or the descending thoracic aorta). The distal extension of the stent graft was based on the distal extension of the dissected aortic aneurysm.

Since 2017 , in addition to TEVAR, we have added a bare stent deployment in the thoraco-abdominal aorta (the Zenith dissection endovascular stent (ZDES), Cook Medical, Bloomington, IN) to induce remodeling of the distal dissected aorta (stent-assisted balloon-induced intimal disruption and relamination in aortic dissection repair-STABILISE technique) $[11,12]$. This technique was chosen when anatomical criteria were favorable (maximum aortic diameter less than 42 $\mathrm{mm})$.

The diameter of the stent graft was sized based on the proximal and distal sealing zone with a maximum $20 \%$ of oversizing compared with the native aorta or ascending aortic graft.

Cerebrospinal fluid drainage (CSFD) was performed when there was extensive coverage of the thoracic aorta $(>250$ $\mathrm{mm})$. Spinal drains were placed preoperatively by anesthesiologists, a small (14-gauge) epidural catheter was placed using anatomic landmarks, with needle placement at L3-L4, and the catheter was advanced $10 \mathrm{~cm}$ after entering the dura. Antiplatelets were stopped 5 days earlier. Spinal fluid was drained to obtain a spinal fluid pressure $<10 \mathrm{mmHg}$ before device deployment.

\section{Radiological Analysis}

All patients were followed up with a postoperative $\mathrm{CT}$ scan before discharge and then at 3 and 6 months and then annually. The preoperative CT scan (after type A repair, before HR for aneurysmal degeneration) and the last CT scan during follow-up were analyzed using three-dimensional imaging software (OSIRIX software, Pixmeo SARL, Geneva, Switzerland). Diameter measurements were performed on the perpendicular axis according to the centerline using a semiautomated centerline algorithm.

Technical success was defined as the exclusion of the lesion, without a type I or III endoleak on perioperative digital subtraction angiography and without perioperative death or surgical conversion.

An unfavorable anatomical outcome was defined as an increase in maximum aortic diameter more than $5 \mathrm{~mm}$, a partial false lumen (FL) thrombosis in the thoracic aorta, a 
Fig. 1 Hybrid repair of residual aortic dissection with redo replacement of the ascending aortic segment in cases of zone 0 debranching. a Preoperative CT scan showing graft angulation that did not provide a good proximal landing zone. b Postoperative CT scan showing the proximal landing zone after redo replacement of the ascending segment and after TEVAR. c Preoperative CT scan showing short ascending replacement that did not provide a good proximal landing zone. d Postoperative CT scan showing elongation of ascending graft after redo replacement of the ascending segment and after TEVAR. The white arrow shows graft angulation. The red arrow shows the distal anastomosis of the ascending aortic graft

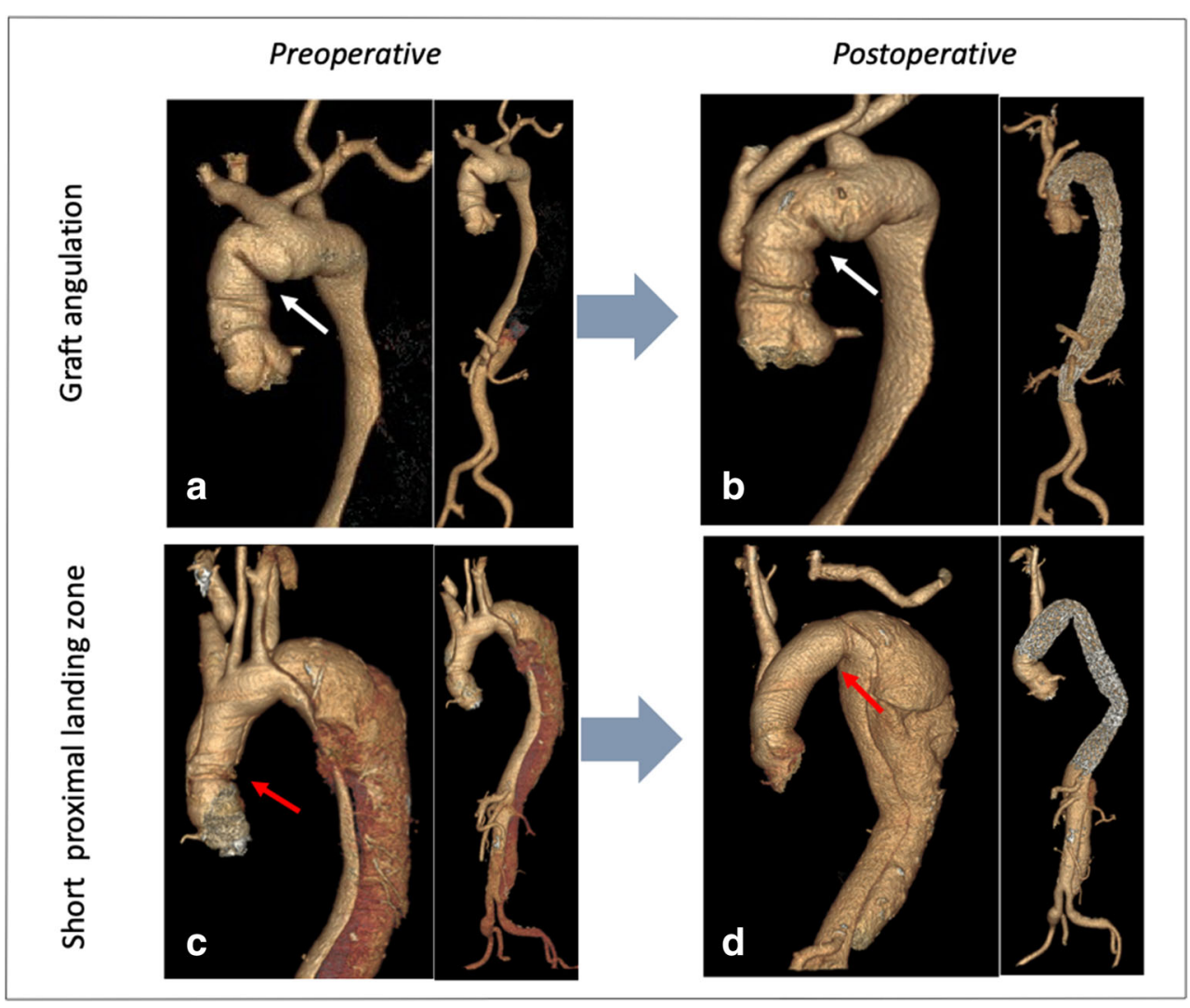

persistent patent FL, or a type I or III endoleak on the postoperative CT scan.

\section{Statistical Analysis}

Data are presented as the mean \pm standard deviation for continuous variables and as counts (\%) for categorical data. The relationship between categorical variables was studied using the chi-square test or Fisher's exact test, when appropriate. The Mann-Whitney $U$ test was used for continuous variables. Normal distribution of the variables was assessed with the Shapiro-Wilk test. All statistical analyses were done using IBM SPSS Statistics 20.0 (IBM Inc., New York, USA).
The probability of survival without distal reinterventions was determined according to the time to last follow-up, including the $95 \%$ confidence intervals (CI), and obtained using Kaplan-Meier estimates. All statistical tests were two-sided, and $P$ values $<0.05$ were considered to indicate statistical significance.

\section{Results}

\section{Demographic Data}

Between November 2009 and January 2020, 529 patients were treated for a type A aortic dissection in our aortic center.
Fig. 2 Hybrid repair of residual aortic dissection in cases of previous IA debranching during previous type A repair. a Control CT scan showing intra-thoracic debranching. b Control CT scan showing extra-thoracic debranching with carotid-tocarotid bypass. The white arrow shows the left common carotid artery reimplantation
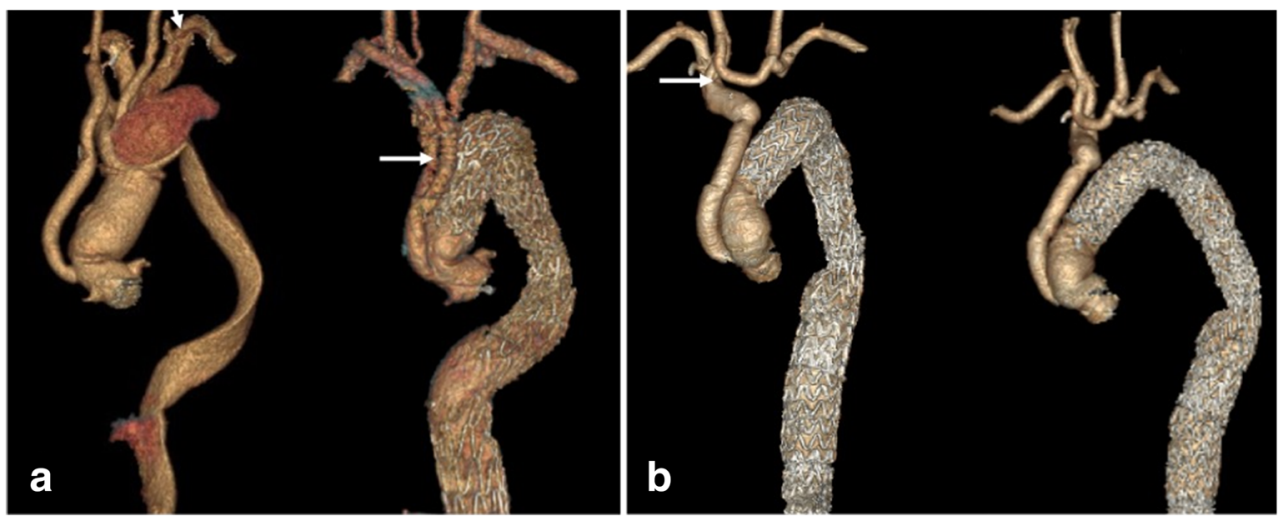
Fig. 3 Hybrid repair of residual aortic dissection that did not involve the aortic arch: left subclavian artery (LSA) debranching alone with endoprosthesis implantation in zone 2. a Preoperative CT scan showing that RAD did not involve the aortic arch. b Control CT scan showing LSA debranching and TEVAR in zone

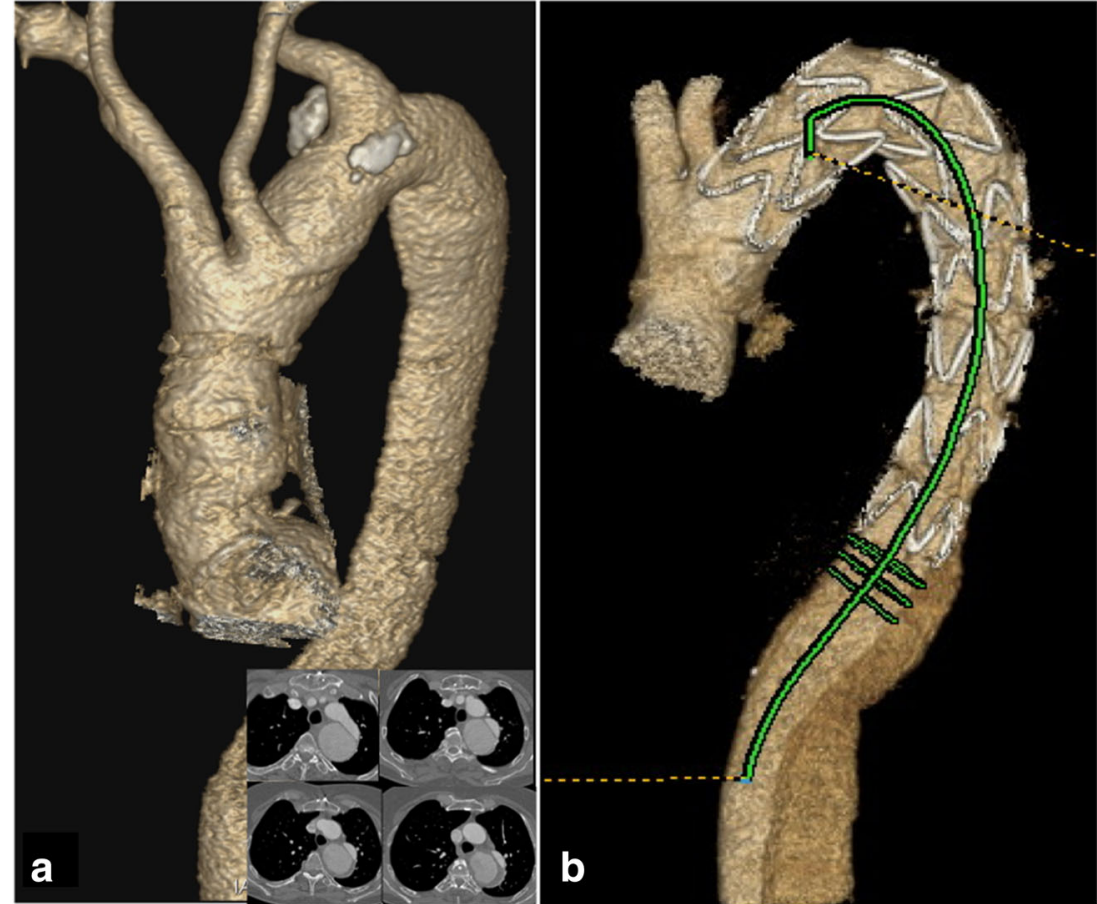

We performed $29 \mathrm{HR}$ for chronic RAD for aneurysmal degeneration of the descending thoracic aorta. Two patients were treated emergently: 1 for aortic rupture and 1 for severe thoracic pain with an aneurysm of $60 \mathrm{~mm}$. The patient's mean age was 59.4 years (range 38 to 78 ). There were 22 men $(75.9 \%)$, and the characteristics of the patients and the first surgical procedures are shown in Table 1.

\section{Debranching Procedure (Table 2)}

The mean delay between the first intervention (type A aortic dissection repair) and HR was 43.8 months (range 3-193).

Twenty-four patients were treated with a complete SA debranching in zone 0.

Five patients had LSA debranching alone and endoprosthesis implantation in zone 2.

\section{Zone 0 Debranching with CPB and CA}

Most of the patients had a previous short ascending aortic replacement or a hemiarch replacement with a residual angulation (Fig. 1a-b), which may compromise a good proximal landing zone. CPB with CA and selective ACP was necessary in 13 patients to ensure a good proximal landing zone for the TEVAR (Fig. 1c-d). Central cannulation with cerebral protection was performed with ACP: The right axillary artery was perfused in 9 patients $(69.2 \%)$, IA in $2(15.3 \%)$, LCCA in 1 $(7.7 \%)$, and right axillary artery with the LCCA in $1(7.7 \%)$. The durations of $\mathrm{CPB}$, aortic cross clamp time, and $\mathrm{CA}$ are reported in Table 2.

\section{Zone 0 Debranching Without CPB and CA}

Three patients had a partial lateral aortic cross clamp.

In 8 cases of previous IA debranching, we performed intrathoracic debranching without CPB in 4 patients and extrathoracic debranching with an intercarotid prosthetic bypass and LSA reimplantation in 4 (Fig. 2a-b).

\section{Zone 2 Debranching}

Among 5 cases of zone 2 debranching, 2 had a total aortic arch replacement during the initial repair. Additionally, in 3 patients, the RAD did not involve the aortic arch. In these 3 cases, patients benefited from a hemiarch replacement during initial type A repair. Moreover, on the postoperative CT scan, there was a RAD beginning after the LSA without RAD on the aortic arch (Fig. 3a-b).

\section{TEVAR}

In 25 patients, a staged procedure was chosen, whereas a concomitant TEVAR was performed in 4 patients (1 rupture, 3 elective). The mean delay between the 2 procedures was 52.1 days (0-214 days). The mean proximal landing zone diameter was $32.6 \mathrm{~mm}$ (28-40), the mean proximal stent graft diameter was $37.7 \mathrm{~mm}(32-45 \mathrm{~mm})$, the mean aortic coverage was $239.5 \mathrm{~mm}$ (115-340), and the mean oversizing was $15.6 \%$ (10-25). Since 2017, we have treated 7 patients with the STABILISE technique when the maximum aortic diameter 
Table 1 Baseline characteristics

\begin{tabular}{ll}
\hline Demographic data & $n=29$ \\
\hline Male sex, $n(\%)$ & $22(75.9)$ \\
Age, mean (SD) & $59.4(9.6)$ \\
Hypertension, $n(\%)$ & $24(82.8)$ \\
Dyslipidemia, $n(\%)$ & $5(17.2)$ \\
Smokers, $n(\%)$ & $14(48.3)$ \\
Obesity, $n(\%)$ & $2(6.9)$ \\
Atrial fibrillation, $n(\%)$ & $3(10.3)$ \\
Coronary artery disease, $n(\%)$ & $4(13.8)$ \\
Renal failure, $n(\%)$ & $4(13.8)$ \\
Marfan syndrome, $n(\%)$ & $4(13.8)$ \\
Indications, $n(\%)$ & \\
Aneurysmal evolution $(>10$ mm) & $28(96.6)$ \\
Rupture & $1(3.4)$ \\
Aortic maximal diameter, mean (SD) & $62.5(10.8)$ \\
Past history of type A aortic repair & \\
Aortic arch replacement, $n(\%)$ & \\
Total aortic arch replacement & $2(6.9)$ \\
Partial aortic arch replacement & $7(24.1)$ \\
Ascending aortic or hemiarch replacement & $20(69.0)$ \\
Aortic root management, $n(\%)$ & $4(13.8)$ \\
Bentall intervention & $25(86.2)$ \\
Supra coronary aortic replacement & \\
\hline
\end{tabular}

Partial arch replacement: replacement between the innominate artery and left subclavian artery. Hemiarch replacement: ascending replacement with open distal anastomosis during moderate hypothermic circulatory arrest with cardiopulmonary bypass, including the lesser curvature of the arch (rather than being limited to the ascending aorta)

$S D$ standard deviation

on the thoraco-abdominal aorta was less than $42 \mathrm{~mm}$. We performed a CFD in 8 cases.

\section{In-Hospital Morbidity and Mortality}

Perioperative morbi-mortality is described in Table 3 according to zone 0 or zone 2 debranching.

Technical success was achieved in $96.5 \%$ (28/29) of cases: There was one perioperative death $(3.4 \%)$ in a patient treated for rupture of a chronic RAD. Among patients treated with complete SA debranching, the mortality rate was $4.2 \%(1 / 24)$.

There was no spinal cord ischemia (SCI), 1 minor stroke after TEVAR (3.4\%) with complete recovery, 1 myocardial infarct $(3.4 \%)$, and 6 pulmonary complications (20.7\%). Two patients had bleeding complications secondary to spinal drains. One patient had a medullar hematoma with cauda equina syndrome (loss of bowel and bladder function without leg paralysis). Another one had a subdural hematoma with complete recovery after neurosurgical evacuation. These patients were on anticoagulation due to a previous mechanic
Table 2 Distribution of patients according to the proximal landing zone and peroperative data

\begin{tabular}{ll}
\hline Population & $n=29$ \\
\hline Zone 0 debranching with CPB and circulatory arrest, $n(\%)$ & $13(44.8)$ \\
$\quad$ Euroscore 2, mean (SD) & $10.7(5.5)$ \\
CPB time (min), mean (SD) & $175(59)$ \\
Aortic cross clamp time (min), mean (SD) & $100(50)$ \\
Circulatory arrest time (min), mean (SD) & $40(17)$ \\
Partial aortic arch replacement, $n(\%)$ & $13(55.544 .8)$ \\
Aortic root management & $2(7.46 .9)$ \\
$\quad$ Bentall, $n$ (\%) & $11(48.137 .9)$ \\
$\quad$ Supra coronary aortic replacement, $n(\%)$ & \\
& $11(37.9)$ \\
Zone 0 debranching without CPB and circulatory arrest, $n(\%)$ & $3(27.210 .3)$ \\
Partial lateral aortic cross clamp, n (\%) & \\
Previous IA debranching * & $4(36.413 .8)$ \\
$\quad$ Intra-thoracic debranching, $n(\%)$ & $4(36.413 .8)$ \\
$\quad$ Extra-thoracic debranching, $n(\%)$ & $5(17.2)$ \\
\hline
\end{tabular}

Extra-thoracic debranching: LCCA and LSA debranching from right common carotid artery through bilateral cervicotomy

$C P B$ cardiopulmonary bypass; $I A$ innominate artery; $S D$ standard deviation

*Intra-thoracic debranching: left common carotid artery (LCCA) and left subclavian artery (LSA) debranching from IA through median sternotomy

Bentall intervention. The rate of minor complications was 27.6\% (8/29): 4 patients with recurrent laryngeal nerve paralysis ( 2 patients with complete and 2 patients with partial recovery), 3 patients with bleeding complications, and 1 patient with delayed healing.

\section{Late Outcomes}

After a median follow-up of 25.4 months (range 3-97 months), the rate of long-term mortality was $10.3 \%(3 / 29)$ with no late aortic-related death; 1 patient died at 9 months due to massive hemorrhagic stroke, and 1 patient died at 97 months due to pneumonia. Complete FL thrombosis of the descending thoracic aorta developed in 27 patients $(93.1 \%)$; the rate of aortic reintervention was $34.5 \%$ (10/29): One patient $(3.4 \%)$ developed a type II endoleak from the LSA at 2 months treated successfully by embolization, 8 patients $(27.6 \%)$ had a reintervention on the thoraco-abdominal aorta, and 1 patient $(3.4 \%)$ had a proximal reintervention (Bentall intervention) (Table 4). The rate of distal aneurysmal progression was $41.4 \%(12 / 29)$. There were 4 patients (13.7\%) with Marfan syndrome, all of whom presented with a distal aortic expansion. In 7 patients treated with STABILISE technique, 6 had a complete aortic remodeling with a thrombosed of the FL on the thoracic aorta and complete reapposition of the FL with relamination of the intimal flap on thoraco-abdominal aorta. 
Table 3 Perioperative morbi-mortality according to zone 0 or zone 2 debranching

\begin{tabular}{|c|c|c|c|}
\hline & \multicolumn{2}{|l|}{ Zone 0 debranching } & \multirow{2}{*}{$\begin{array}{l}\text { Zone } 2 \text { debranching } \\
\text { Partial debranching }(n=5)\end{array}$} \\
\hline & With CPB and CA $(n=13)$ & Without CPB and CA $(n=11)$ & \\
\hline Technical success, $n(\%)$ & $12(92.3)$ & $11(100)$ & $5(100)$ \\
\hline Mortality, $n(\%)$ & $1(7.7)$ & $0(0)$ & $0(0)$ \\
\hline Spinal cord ischemia, $n(\%)$ & $0(0)$ & $0(0)$ & $0(0)$ \\
\hline Stroke, $n(\%)$ & $0(0)$ & $1(9)$ & $0(0)$ \\
\hline Myocardial infarct, $n(\%)$ & $1(7.7)$ & $0(0)$ & $0(0)$ \\
\hline Pneumonia, $n(\%)$ & $6(46.1)$ & $0(0)$ & $0(0)$ \\
\hline
\end{tabular}

$C P B$ cardiopulmonary bypass; $C A$ circulatory arrest

The Kaplan Meier estimated freedom for aortic reintervention or death at 2 years and 5 years was $73 \%$ and $66.5 \%$, respectively (Fig. 4).

\section{Risk Factors for Distal Aortic Aneurysmal Degeneration and Reinterventions}

Risk factors for distal aneurysmal degeneration were delay between acute type A repair and HR (73.1 vs. 28.9 months, $P=0.068$ ), the initial maximal aortic diameter (71.7 vs. 59.1 $\mathrm{mm}, P<0.01)$, and the presence of a distal new entry tear (45.4\% (5/11) vs. $11.1 \%(2 / 18), P=0.07)$. Risk factors for distal aortic reintervention were delay between acute type A repair and HR ( 82.8 vs. 31.4 months, $P=0.014$ ), the initial maximal aortic diameter (75.1 vs. 59.8, $P<0.01)$, and the presence of a distal new entry tear $(57.1 \%(4 / 7)$ vs. $13.6 \%$ (3/22), $P=0.038)$ (Table 5).

There was $14.2 \%$ (1/7) distal aneurysmal degeneration on thoraco-abdominal aorta in the STABILISE group compared to $45.5 \%(10 / 22)$ in the TEVAR alone group $(P=0.20)$ and none distal reintervention $(P=0.14)$. Proximal landing zone management did not influence late outcomes and risk of distal aneurysmal degeneration and reinterventions.

\section{Comment}

We report here an observational study with interesting results because it is the largest study on HR in chronic RAD. There are no guidelines regarding the treatment of distal aneurysmal degeneration of chronic RAD. Open surgery with total arch replacement, frozen elephant trunk (FET), hybrid treatment with supra-aortic trunk debranching and TEVAR, or total endovascular solution with fenestrated/branched endoprosthesis are different options [13].

Open aortic arch surgery with prior ascending aortic replacement is challenging and is associated with a high rate of mortality, ranging from 5 to $20 \%$ [14-17]. In a recent study,

Table 4 Long-term reoperations

\begin{tabular}{lllll}
\hline $\begin{array}{l}\text { Zone implantation } \\
\text { endoprosthesis }\end{array}$ & Delay, days & Reason & Reintervention & Perioperative morbi-mortality \\
\hline 0 & 84 & Distal evolution & RV debranching + TEVAR & 0 \\
0 & 124 & Distal evolution & RV debranching + TEVAR & 0 \\
0 & 113 & Distal evolution & RV debranching + TEVAR & Pulmonary complications \\
0 & 2239 & Distal evolution & FEVAR & 0 \\
2 & 184 & Distal evolution & FEVAR & Retroperitoneal hematoma \\
0 & 329 & Distal evolution & EVAR & 0 \\
0 & 67 & Distal evolution & Aorto-bifemoral bypass graft * & 0 \\
0 & 783 & Proximal evolution & Bentall & Pulmonary complications \\
0 & 67 & Type II endoleak & LSA embolization & 0 \\
0 & 63 & Distal evolution & Aorto-biiliac bypass graft * & 0 \\
\hline
\end{tabular}

RV debranching: reno-visceral debranching from the common iliac artery

FEVAR fenestrated endovascular aortic repair; EVAR endovascular aortic repair; TEVAR thoracic endovascular aortic repair; LSA left subclavian artery

*Aneurysmal degeneration limited to infrarenal abdominal aorta 
Fig. 4 Long-term outcomes: Kaplan-Meier event-free survival curve for distal aortic reintervention or death (aortic events) after TEVAR for residual aortic dissection (+: censored data). $* \mathrm{SE}>10 \%$

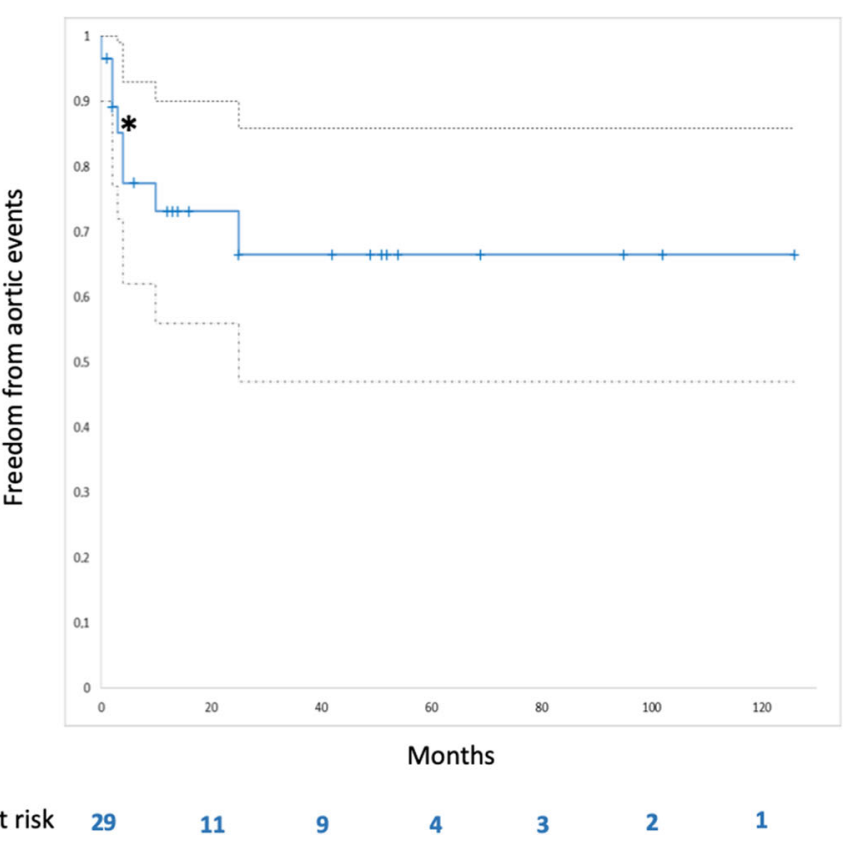

among 117 reoperations after acute type A aortic dissection repair, Dell'Aquila et al. reported an in-hospital mortality of $19.6 \%$. Furthermore, 31 patients underwent a distal reoperation ( $61.2 \%$ of total arch reoperation and $22.5 \%$ of FET), with an in-hospital mortality of $25.8 \%$ [14].

Total endovascular aortic arch repair with branched endograft, avoiding open reoperation, may be a good option with a potentially low stroke and death rate (4\%), as shown in a recent study published by Verscheure et al. [18]. Currently, these devices and techniques are investigational, limited to a few centers, with results to be duplicated on a larger scale.

Our study reports the results of hybrid arch repair specifically for chronic RAD and confirmed that hybrid treatment could be a safe and effective technique with a low rate of inhospital mortality and morbidity relative to historical controls. The rate of in-hospital mortality after hybrid aortic arch repair varies from 3 to $30 \%$ in several studies [19-21], correlated with the number of arterial debranching procedures performed, proximal landing zone, and type of pathology treated.
The present study is one of the larger studies that included only patients with chronic RAD [22], with a mortality rate of $4.2 \%$ $(1 / 20)$ in patients with zone 0 debranching, far below the mortality rates of $20-40 \%$ found in other series [21, 23, 24].

The rate of neurologic complications can also be very significant in these challenging cases [25]. In a case series published in 2016 by Canaud et al., the authors reported 2 strokes and $1 \mathrm{SCI}$ among 7 hybrid arch repairs for chronic RAD [22]. In our cohort, there was only 1 stroke and no paraplegia. We believe that systematic reimplantation of the LSA is protective $[10,23]$, and CSFD performed when there is extensive coverage of the thoracic aorta $(>250 \mathrm{~mm})$ reduces the rate of spinal cord complications. However, we did observe 2 complications linked to CSFD in patients with therapeutic anticoagulation; CSFD should probably be used with care in these cases.

In 13 cases, we opted for a redo replacement of the ascending aorta, when the previous ascending replacement during type A repair did not provide a good proximal landing zone

Table 5 Risk factors for distal aneurysmal degeneration and reinterventions

\begin{tabular}{|c|c|c|c|}
\hline & Aneurysmal degeneration $n=11$ & No aneurysmal degeneration $n=18$ & $P$ value \\
\hline Delay between type A repair and HR (months), mean (SD) & $73.1(67.4)$ & $28.9(32.4)$ & 0.068 \\
\hline Initial maximum aortic diameter (mm), mean (SD) & $71.7(11.8)$ & $590(8.8)$ & $<0.01$ \\
\hline \multirow[t]{2}{*}{ Distal new entry tear, $n(\%)$} & $5(45.4)$ & $2(11.1)$ & 0.07 \\
\hline & Distal reinterventions $n=7$ & No distal reinterventions $n=22$ & $P$ value \\
\hline Delay between type A repair and HR (months), mean (SD) & $82.8(55.9)$ & $31.4(46.7)$ & 0.014 \\
\hline Initial maximum aortic diameter (mm), mean (SD) & $75.1(12.6)$ & $59.7(8.4)$ & $<0.01$ \\
\hline Distal new entry tear, $n(\%)$ & $4(57.1)$ & $3(13.6)$ & 0.038 \\
\hline
\end{tabular}

$H R$ hybrid repair; $S D$ standard deviation 
(angulation or short replacement) and the residual dissection involved the aortic arch. In our experience, this approach allows for a safer proximal landing zone and significantly reduces the risk of type 1 endoleak after TEVAR. With a technical success rate of $96.4 \%$, the absence of in-hospital mortality in elective patients and the absence of type I endoleak, this study showed encouraging early results that could be explained by a complete and multidisciplinary preoperative evaluation, the use of ACP systematically in case of CA and the vast experience of our aortic center. Moreover, HR provides a less invasive option in this specific setting by limiting the extent of surgical intervention on the aortic arch, reducing the duration of the CA and perioperative mortality [25].

However, despite good anatomical results in stabilizing the proximal part of the thoracic aorta, we observed aneurysmal progression of the thoraco-abdominal aorta in about half of the patients, requiring aortic reintervention in most cases. Faure et al. proposed the STABILISE technique to avoid these negative evolutions $[11,26]$. In our series, there were no major complications related to this technique, and the STABILISE technique seems to be associated with good anatomical results. We identified a delay between type A aortic dissection and HR as a risk factor of distal aneurysmal degeneration and aortic reinterventions. Indeed, the chronicity of the dissection has relevance with regard to aortic remodeling after endovascular therapy, which is significantly greater in patients with acute or subacute dissection. Endovascular repair of acute or subacute dissection is associated with rapid expansion of the true lumen and collapse of the FL. In contrast, endovascular treatment for chronic dissection can induce FL thrombosis in the treated segment without a change in the aortic diameter in most cases and with a patent FL on thoraco-abdominal aorta [27]. This also links to distal new entry tears identified in the present study as risk factors for distal aneurysmal degeneration and reinterventions. Stent graft-induced new entry is a well-known risk factor for aneurysm development and is more common in chronic aortic dissection and when the distal oversizing of the graft stent is $>20 \%$ [28].

Finally, initial aortic diameter was also significatively associated with an increased risk of aneurysmal degeneration and reintervention. This suggests that aortic dissection is a pathology of the whole aorta. The more severe the disease is at the thoracic level, the more severe it is at the thoraco-abdominal level.

\section{Limitations}

The small size and retrospective nature of our study limits the interpretation of the results. Moreover, these results were observed at one aortic center, which can introduce a possible confounding bias. Moreover, the lack of a comparison arm is a limitation of this study, but we cannot provide a comparative study because HR remains our first-line therapy.

\section{Conclusion}

HR of RAD in a high-volume center is associated with good anatomical results and an acceptable risk of perioperative morbi-mortality, including for patients treated in zone 0 . As a less invasive approach compared to conventional surgery, this treatment provides an alternative. A redo replacement of the ascending aortic segment is necessary when the previous ascending replacement during type A repair does not provide a good proximal landing zone (angulation or short replacement) to reduce the risk of type I endoleak after TEVAR.

Supplementary Information The online version contains supplementary material available at https://doi.org/10.1007/s10557-021-07150-w.

Acknowledgements We would like to thank Professor Michel S. Makaroun (Pittsburgh, PA) for his careful reading and comments.

Author Contribution All authors contributed to the study conception and design. Material preparation, data collection, and analysis were performed by Alizée Porto, Arnaud Blanchard, Jean Victor Chazot, Laurence Bal, Mariangela De Masi, Pierre-Antoine Barral, Alexis Jacquier, Vlad Gariboldi, Frederic Collart, Valerie Deplano, and Marine Gaudry. The first draft of the manuscript was written by Marine Gaudry, Philippe Piquet, and Alizee Porto. Axel Bartoli made all figure revisions needed to improve the manuscript. All authors commented on previous versions of the manuscript. All authors read and approved the final manuscript.

Availability of Data and Material All the patients included in this study were clearly informed about the use of their data for clinical research, and the institutional review board approved the project (approval number 2019-48).

Code Availability Not applicable.

\section{Declarations}

Ethical Approval All procedures performed in studies involving human participants were in accordance with the ethical standards of the institutional research committee and with the 1964 Helsinki Declaration and its later amendments or comparable ethical standards.

Informed Consent Informed consent was obtained from all participants included in the study.

Conflict of Interest The authors declare no competing interests.

\section{References}

1. Concistre G, Casali G, Santaniello E, et al. Reoperation after surgical correction of acute type A aortic dissection: risk factor analysis. Ann Thorac Surg. 2012;93:450-5.

2. Zierer A, Voeller RK, Hill KE, Kouchoukos NT, Damiano RJ Jr, Moon MR. Aortic enlargement and late reoperation after repair of acute type A aortic dissection. Ann Thorac Surg. 2007;84:479-86. discussion 486-7. 
3. Tamura K, Chikazawa G, Hiraoka A, Totsugawa T, Sakaguchi T, Yoshitaka $\mathrm{H}$. The prognostic impact of distal anastomotic new entry after acute type I aortic dissection repair. Eur J Cardiothorac Surg. 2017;52:867-73.

4. Fattouch K, Sampognaro R, Navarra E, Caruso M, Pisano C, Coppola $\mathrm{G}$, et al. Long-term results after repair of type A acute aortic dissection according to false lumen patency. Ann Thorac Surg. 2009;88:1244-50.

5. Kimura N, Itoh S, Yuri K, Adachi K, Matsumoto H, Yamaguchi A, et al. Reoperation for enlargement of the distal aorta after initial surgery for acute type A aortic dissection. J Thorac Cardiovasc Surg. 2015;149:S91-8. e1.

6. Nakamura K, Onitsuka T, Yano M, Yano Y, Matsuyama M, Furukawa K. Risk factor analysis for ascending aorta and aortic arch repair using selective cerebral perfusion with open technique: role of open-stent graft placement. J Cardiovasc Surg. 2006;47: 659-65.

7. Kazui T, Yamashita K, Washiyama N, et al. Aortic arch replacement using selective cerebral perfusion. Ann Thorac Surg. 2007;83: S796-8. discussion S824-31.

8. Haulon S, Greenberg RK, Spear R, Eagleton M, Abraham C, Lioupis $\mathrm{C}$, et al. Global experience with an inner branched arch endograft. J Thorac Cardiovasc Surg. 2014;148:1709-16.

9. Koullias GJ, Wheatley GH 3rd. State-of-the-art of hybrid procedures for the aortic arch: a meta-analysis. Ann Thorac Surg. 2010;90:689-97.

10. Cao P, De Rango P, Czerny M, et al. Systematic review of clinical outcomes in hybrid procedures for aortic arch dissections and other arch diseases. J Thorac Cardiovasc Surg. 2012;144:1286-300. 1300 e1-2.

11. Faure EM, El Batti S, Sutter W, et al. Stent-assisted balloon-induced intimal disruption and relamination of distal remaining aortic dissection after acute DeBakey type I repair. J Thorac Cardiovasc Surg. 2019;157:2159-65.

12. Faure EM, El Batti S, Sutter W, et al. Stent-assisted balloon dilatation of chronic aortic dissection. J Thorac Cardiovasc Surg. 2020;S0022-5223(20)30430-X. Online ahead of print.

13. Czerny M, Kreibich M, Morlock J, Kondov S, Scheumann J, Schröfel H, et al. Chronic type B "residual" after type A: what I would do? J Vis Surg. 2018;4:14

14. Dell'Aquila AM, Pollari F, Fattouch K, Santarpino G, Hillebrand J, Schneider S, et al. Early outcomes in re-do operation after acute type A aortic dissection: results from the multicenter REAAD database. Heart Vessel. 2017;32:566-73.

15. Malvindi PG, van Putte BP, Sonker U, Heijmen RH, Schepens MA, Morshuis WJ. Reoperation after acute type A aortic dissection repair: a series of 104 patients. Ann Thorac Surg. 2013;95:922-7.

16. Quintana E, Bajona P, Schaff HV, Dearani JA, Daly RC, Greason $\mathrm{KL}$, et al. Open aortic arch reconstruction after previous cardiac surgery: outcomes of 168 consecutive operations. J Thorac Cardiovasc Surg. 2014;148:2944-50.
17. Di Bartolomeo R, Berretta P, Pantaleo A, et al. Long-term outcomes of open arch repair after a prior aortic operation: our experience in 154 patients. Ann Thorac Surg. 2017;103:1406-12.

18. Verscheure D, Haulon S, Tsilimparis N, Resch T, Wanhainen A, Mani K, et al. Endovascular treatment of post type A chronic aortic arch dissection with a branched endograft: early results from a retrospective international multicenter study. Ann Surg. 2019; Online ahead of print.

19. Papakonstantinou NA, Antonopoulos CN, Baikoussis NG, Kakisis I, Geroulakos G. Aortic arch reconstruction: are hybrid debranching procedures a good choice? Heart Lung Circ. 2018;27:1335-49.

20. Shirakawa Y, Kuratani T, Shimamura K, Torikai K, Sakamoto T, Shijo T, et al. The efficacy and short-term results of hybrid thoracic endovascular repair into the ascending aorta for aortic arch pathologies. Eur J Cardiothorac Surg. 2014;45:298-304. discussion 304.

21. Antoniou GA, El Sakka K, Hamady M, Wolfe JH. Hybrid treatment of complex aortic arch disease with supra-aortic debranching and endovascular stent graft repair. Eur J Vasc Endovasc Surg. 2010;39:683-90.

22. Canaud L, Gandet T, Ozdemir BA, Albat B, Marty-Ane C, Alric P. Hybrid aortic repair of dissecting aortic arch aneurysm after surgical treatment of acute type A dissection. Ann Vasc Surg. 2016;30:17580.

23. Antoniou GA, Mireskandari M, Bicknell CD, Cheshire NJW, Gibbs RG, Hamady M, et al. Hybrid repair of the aortic arch in patients with extensive aortic disease. Eur J Vasc Endovasc Surg. 2010;40:715-21.

24. Vallejo N, Rodriguez-Lopez JA, Heidari P, Wheatley G, Caparrelli $\mathrm{D}$, Ramaiah $\mathrm{V}$, et al. Hybrid repair of thoracic aortic lesions for zone 0 and 1 in high-risk patients. J Vasc Surg. 2012;55:318-25.

25. Conzelmann LO, Hoffmann I, Blettner M, Kallenbach K, Karck M, Dapunt $\mathrm{O}$, et al. Analysis of risk factors for neurological dysfunction in patients with acute aortic dissection type A: data from the German Registry for Acute Aortic Dissection type A (GERAADA). Eur J Cardiothorac Surg. 2012;42:557-65.

26. Faure EM, El Batti S, Abou Rjeili M, Julia P, Alsac JM. Mid-term outcomes of stent assisted balloon induced intimal disruption and relamination in aortic dissection repair (STABILISE) in acute type B aortic dissection. Eur J Vasc Endovasc Surg. 2018;56:209-15.

27. Sayer D, Bratby M, Brooks M, Loftus I, Morgan R, Thompson M. Aortic morphology following endovascular repair of acute and chronic type B aortic dissection: implications for management. Eur J Vasc Endovasc Surg. 2008;36(5):522-9.

28. Canaud L, Gandet T, Sfeir J, Ozdemir BA, Solovei L, Alric P. Risk factors for distal stent graft-induced new entry tear after endovascular repair of thoracic aortic dissection. J Vasc Surg. 2019;69(5):1610-4.

Publisher's Note Springer Nature remains neutral with regard to jurisdictional claims in published maps and institutional affiliations. 